\section{Eco-evolutionary modeling of planetary ecosystems to predict habitability and biosignatures - the cases of early Earth and Mars} BORIS SAUTEREY ${ }^{1}$, ANTONIN AFFHOLDER $^{1,2}$,
FRANCOIS J GUYOT $^{3}$, BENJAMIN CHARNAY
STÉPHANE MAZEVET $^{2}$ AND RÉGIS FERRIÈRE

${ }^{1}$ Institut de Biologie de l'École Normale Supérieure

${ }^{2}$ Observatoire de Paris

${ }^{3}$ CNRS-Institut de Minéralogie, de Physique des Matériaux et de Cosmochimie, Sorbonne Université - CNRS - MNHN

${ }^{4}$ Department of Ecology and Evolutionary Biology

Presenting Author: boris.sauterey@biologie.ens.fr

Models of planetary ecosystem have played a major role in elucidating how our planet's biosphere has shaped Earth's key characteristics throughout its history (e.g., atmospheric composition, climate) and helped interpreting geological evidence to constrain the evolutionary history of life on Earth $[1,2,3]$. These models can be extended beyond Earth to help predict planetary biosignatures that could be used to search for signs of life on other planets, given current or foreseeable technologies, and to reduce uncertainties in discriminating lifeless and inhabited planets [4,5].

Existing models can represent abiotic planetary processes in much detail. In contrast, the way they resolve life feedbacks to the planetary environment remains often rudimentary. An oversimplification of biological processes may result in inaccurate predictions regarding planetary habitability and planetary features that might be candidate biosignatures. Here we present a new, thermodynamically-grounded framework to modeling planetary ecosystems which explicitly includes the physiological, ecological and evolutionary processes of chemotrophic microbial biospheres to address both their energetic limits and their feedback to the planetary environment.

We show how this approach can be used to produce refined predictions regarding the co-evolutionary history of the Archean Earth with its primordial chemotrophic biosphere and the role of life in shaping the global atmospheric and climatic characteristics of the planet [6]. Our approach can furthermore be applied to Mars to evaluate its early habitability and to model how the coevolution with a primitive biosphere similar to Earth's feeds back to and reshape Mars' initial habitability. The model predicts likely habitats and their planetary distribution, where such a primitive Martian biosphere might have flourished. Our results could guide ongoing and future explorations of the Martian surface and subsurface (e.g., Mars 2020 exploration of Jezero Crater) in the search for signs of ancient and extant life.

[1] Kasting, J.F. et al. (2001) Origins of Life and Evolution of the Biosphere. [2] Kharecha, P. et al. (2005) Geobiology. [3] Catling, D.C. et al. (2007) PTRS A. [4] Krissanson-Totton, J. et al. (2018) PNAS. [5] Catling, D.C. et al. (2018) Astrobiology. [6] Sauterey, B. et al. (2020) Nat. Comm.

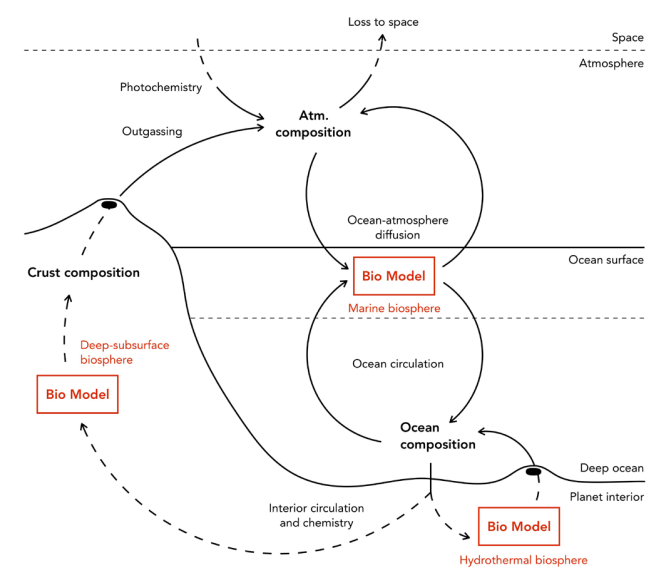

